\title{
Primary Leiomyosarcoma of Bone: A Case Report
}

\author{
Mahdi Hakiminezhad ${ }^{1}$, Maziar Nafisi ${ }^{2}$, Hana Saffar ${ }^{3}$ and Sadegh Saberi ${ }^{2,{ }^{*}}$ \\ ${ }^{1}$ MD, MPH, Students' Scientific Research Center, Tehran University of Medical Sciences, Tehran, IR Iran \\ ${ }^{2}$ MD, Department of Orthopedic surgery, Imam Khomeini Hospital Complex, Tehran University of Medical Sciences, Tehran, IR Iran \\ ${ }^{3}$ MD, Department of Pathology, Imam Hospital Complex, School of Medicine, Tehran University of Medical Sciences, Tehran, IR Iran \\ "Corresponding author: Sadegh Saberi, MD, Department of Orthopedic surgery, Imam Khomeini Hospital Complex, Tehran University of Medical Sciences, Tehran, IR Iran. \\ E-mail: s_saberi@sina.tums.ac.ir
}

Received 2017 May 06; Accepted 2017 June 12.

\begin{abstract}
Leiomyosarcoma is an aggressive type of soft tissue neoplasm that originates from smooth muscle cells; it is typically found in the uterus, gastrointestinal tract, and retroperitoneum. Primary leiomyosarcoma of bone is an extremely rare phenomenon first reported in 1965. Here, we present a case of primary leiomyosarcoma of bone occurred in the proximal humerus of a 41-year-old man who was referred to our orthopaedic clinic with a chief complaint of persistent left shoulder pain. Radiological investigations revealed a proximal lesion of the left humerus with periosteal reaction. Core needle biopsy of the lesion was performed later, which pointed to primary leiomyosarcoma of bone as the underlying pathology. Subsequently, complete resection of the lesion and prosthetic replacement of the proximal humorous were performed. The patient was followed three months after the surgery, and no sign of local recurrence or metastatic lesion was detected. To the best of our knowledge, this is the first case report of primary leiomyosarcoma of bone in Iran.
\end{abstract}

\section{Introduction}

Leiomyosarcoma is an aggressive type of soft tissue neoplasm that originates from smooth muscle cells. It is typically found in the uterus, gastrointestinal tract, and retroperitoneum. Primary leiomyosarcoma of bone is an extremely rare phenomenon, which was first reported in 1965 (1). Due to its rare incidence and unspecific radiologic features, leiomyosarcoma of bone is commonly misdiagnosed with other pleomorphic undifferentiated sarcomas such as osteosarcoma, chondrosarcoma, or other unspecified sarcomas of bone (2). This pathology is predominantly reported in adult males, especially in the distal femur and other large bones. Here, we present a case of primary leiomyosarcoma of bone occurred in the proximal humerus of 41-year-old man. To the best of our knowledge, this is the first case report of primary leiomyosarcoma of bone in Iran.

\section{Case Presentation}

A 41-year-old man was referred to our Hospital with a two-year history of persistent left shoulder pain. His pain could be moderately relieved by taking simple analgesics. No prior history of trauma or recent fever, weight loss, and night sweats were detected and his medical history was otherwise insignificant.
Initial physical examination revealed no sign of local tenderness, swelling, or lymphadenopathy. Motor and sensory functions were intact, and the shoulder range of motion was within the normal limits. Laboratory investigations were normal for complete blood count, electrolytes, erythrocyte sedimentation rate (ESR), and C-reactive protein (CRP). For further evaluation, radiological investigations were performed indicating a proximal lesion of the left humerus with a periosteal reaction. No sign of reactive sclerosis or ossification was evident.

Bone cancer and metastatic tumors were considered the main differential diagnoses. Core needle biopsy of the lesion was performed later and its result was in favour of primary leiomyosarcoma of bone as underlying pathology. Subsequently, complete resection of the lesion and prosthetic replacement of the proximal humorous were performed. The resected tumor was sent to the pathology department for histopathological evaluations. The tumor specimen was a multinodular cream-grayish tissue with elastic consistency attached to humorous head and neck, measuring $6 \mathrm{~cm}$ in maximal diameter (Figure 1). Microscopic examinations showed infiltration of a neoplasm composed of highly atypical large bizarre pleomorphic cells some with moderate amount of cytoplasm arranged in fascicular, storiform, and diffuse pattern (Figure 2). Mitotic figures were frequent with $16 / 10$ high power field mi- 
totic count including atypical forms. The immunohistochemical staining was positive for vimentin, caldesmin, desmin, smooth muscle actin (SMA), alpha 1-antitrypsin, and epithelial membrane antigen. These findings confirmed the diagnosis of primary leiomyosarcoma of proximal humerus in this patient. The patient was followed for 3 months after surgery, and no sign of local recurrence or metastatic lesions were detected.

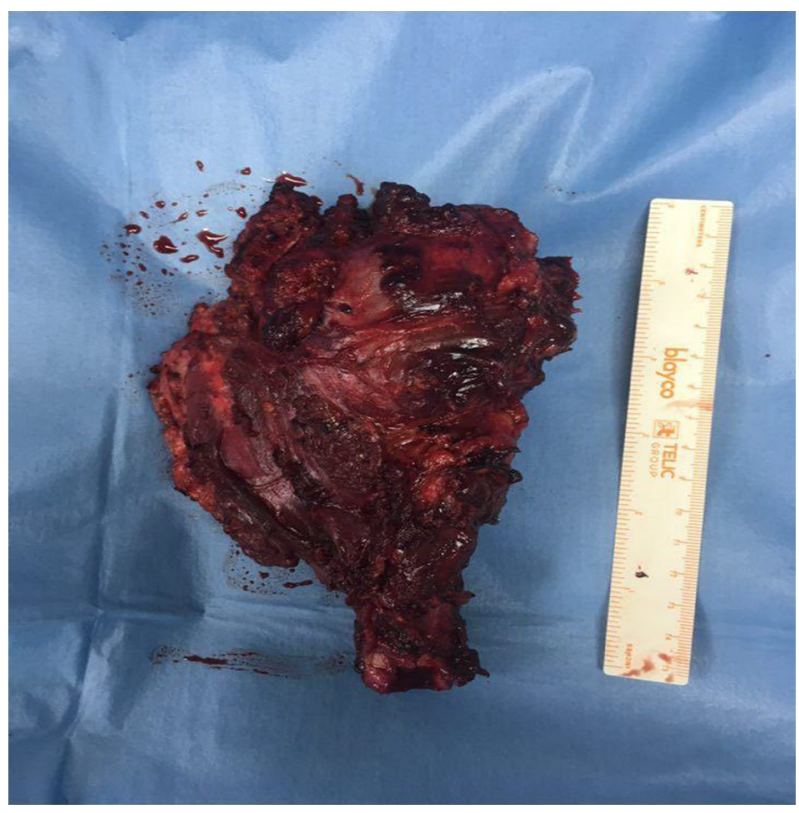

Figure 1. The Resected Tumor Attached to Humorous Head and Neck

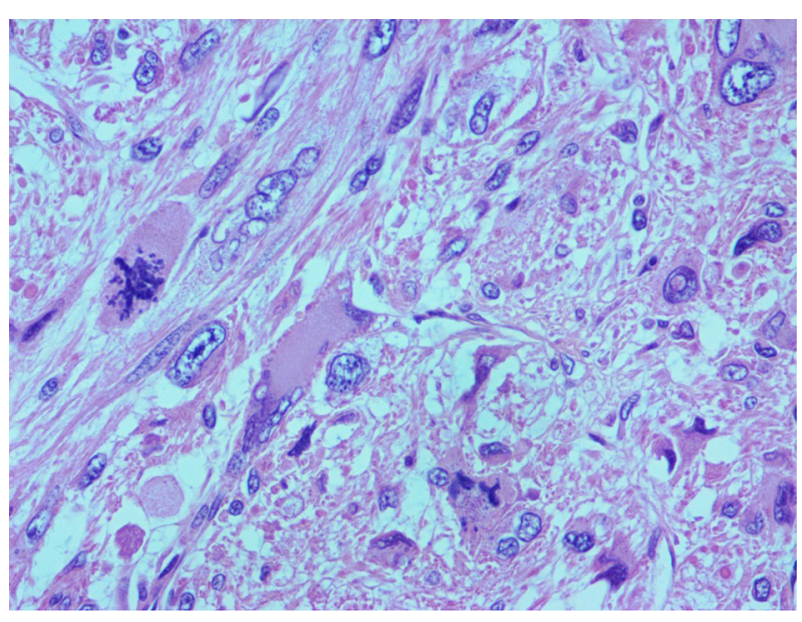

Figure 2. Pathological Hematoxylin and Eosin Staining of the Tumor Tissue of the Resected Primary Leiomyosarcoma of Proximal Humerus

\section{Discussion}

Here we presented a rare case of primary leiomyosarcoma of the proximal humerus in a middle-aged man that was successfully treated by surgical resection of the tumor. Leiomyosarcoma of bone is an uncommon bone neoplasm with a prevalence rate of less than $1 \%$ among all types of malignant bone tumors (3). Persistent pain is the main cause of referral in the affected patients. Regional swelling is common. Pathologic bone fracture has also been reported in these patients (2).

By improvement of diagnostic tests and pathological examinations in recent years, the number of cases diagnosed with primary leiomyosarcoma has increased notably. Characteristic radiographic findings in primary leiomyosarcoma are osteolytic lesion with moth-eaten appearance and indistinct margins (2). Irregular cortical erosions may be apparent, but sclerosis and periosteal reaction are less common (3). Excisional biopsy and Immunohistochemical evaluations are often required for confirming the diagnosis (4).

Major differential diagnoses of the leiomyosarcoma of bone are metastatic carcinoma of unknown primary origin, lymphoma, and osteosclerotic myeloma. It is important to differentiate between leiomyoma and leiomyosarcoma especially when a previous history of benign leiomyoma exists (5).

Surgical resection with a wide margin is the main treatment option for primary leiomyosarcoma of bone. Therapeutic benefits of chemotherapy and radiotherapy are still unclear (3). Local recurrence of the tumor is uncommon, but the risk of distal metastasis is relatively high especially in patients with high-grade tumors (2). Tumor stage is the main predictor of long-term outcome, but it seems that the 5-year overall survival rate of primary leiomyosarcoma of bone is $59 \%$ that is similar to other common bone sarcomas (2).

\section{References}

1. Evans DM, Sanerkin NG. Primary leiomyosarcoma of bone. J Pathol Bacteriol.1965;90(1):348-50. [PubMed: 5843955].

2. Adelani MA, Schultenover SJ, Holt GE, Cates JM. Primary leiomyosarcoma of extragnathic bone: clinicopathologic features and reevaluation of prognosis. Arch Pathol Lab Med. 2009;133(9):1448-56. doi: 10.1043/1543-2165-133.9.1448. [PubMed: 19722754].

3. Brewer P, Sumathi V, Grimer RJ, Carter SR, Tillman RM, Abudu A, et al. Primary leiomyosarcoma of bone: analysis of prognosis. Sarcoma. 2012;2012:636849. doi: 10.1155/2012/636849. [PubMed: 22550421].

4. Antonescu CR, Erlandson RA, Huvos AG. Primary leiomyosarcoma of bone: a clinicopathologic, immunohistochemical, and ultrastructural study of 33 patients and a literature review. Am J Surg Pathol. 1997;21(11):1281-94. [PubMed: 9351566].

5. Yang Y, Ma L, Li L, Liu H. Primary leiomyosarcoma of the spine: A case report and literature review. Medicine (Baltimore). 2017;96(9). e6227. doi: 10.1097/MD.0000000000006227. [PubMed: 28248883]. 